\title{
A facile scalable conductive graphene-coated Calotropis gigantea yarn
}

\author{
Junze Zhang $\cdot$ Jing Liu $\cdot$ Zeyu Zhao $\cdot$ Di Huang $\cdot$ Chao Chen $\cdot$ \\ Zhaozhu Zheng $\cdot$ Chenxi Fu $\cdot$ Xiaoqin Wang $\cdot$ Yanxue Ma $\cdot$ Yuling Li $\cdot$ \\ Zekun Liu $\cdot$ Yi Li $\cdot$ Gang Li
}

Received: 30 August 2021 / Accepted: 8 February 2022 / Published online: 1 March 2022

(C) Crown 2022

\begin{abstract}
Graphene-functionalized fibers have attracted substantial attention due to their potential applications in flexible wearable electronics. However, these conventional conductive materials face difficulties in mass production, which limits their large-scale fabrication. In this paper, we report a graphene-coated Calotropis gigantea yarn by pad dyeing with graphene oxide and a reduction process, which endows it with high conductivity, outstanding conducting stability, and scale production capacity. By optimizing the dyeing parameters, the modified yarns display a high electrical conductivity of $6.9 \mathrm{~S} / \mathrm{m}$. Range analysis results indicate that the electrical conductivity of the graphene-coated yarns exhibits a strong dependence on the concentration of graphene
\end{abstract}

J. Zhang $\cdot$ J. Liu $\cdot$ Z. Zhao $\cdot$ D. Huang $\cdot$ C. Chen $\cdot$

Z. Zheng $\cdot$ C. Fu $\cdot$ X. Wang $\cdot$ G. Li $(\bowtie)$

National Engineering Laboratory for Modern Silk, College of Textile and Clothing Engineering, Soochow University, Suzhou 215123, China

e-mail: tcligang@suda.edu.cn

Y. Ma $\cdot$ Y. Li

Key Laboratory of Textile Science and Technology, Ministry of Education, Donghua University,

Shanghai 201620, China

Z. Liu $(\varangle) \cdot \operatorname{Y} . \operatorname{Li}(\bowtie)$

Department of Materials, University of Manchester, Oxford Road, Manchester M13 9PL, UK

e-mail: zekun.liu@manchester.ac.uk

Y. Li

e-mail: henry.yili@manchester.ac.uk oxide and pad dyeing cycles. The hydrogen bonding between the fiber and graphene during the dyeing process renders the functionalized yarns stable conductivity to washing and bending. Based on the simple fabrication process and fascinating performance, the graphene-coated yarn show great potential in facile scale production of conductive yarns.

Keywords Graphene - Calotropis gigantea . Conductive fibers $\cdot$ Surface coating $\cdot$ Wearable electronics

\section{Introduction}

In recent years, the research about multifunctional flexible wearable electronic textiles in flexible sensors (Liu et al. 2021a; Gandla et al. 2020; Wang et al. 2020; Lu et al. 2020), health indicators (Liu et al. 2021b, c; Won et al. 2020; Zhao et al. 2020), and electromagnetic interference shielding (Ren et al. 2018; Ozen et al. 2018; Jia et al. 2019) has attracted extensive attention due to their great potential as next-generation electronics with the advantages of lightweight, flexibility, and portability, which can be applied for personal motion detection and healthcare, man-machine interaction, artificial intelligence and so forth (Lin et al. 2018). There are many approaches for the preparation of electronic fibers through the modification of polyaniline, metal nanoparticles, carbon nanotubes, graphene, etc. Among the active 
candidates, graphene possesses a unique 2-D sheetlike structure (Geim and Novoselov 2007), with extremely high electrical conductivity, high mechanical strength, and good chemical stability (Molina 2016). It can be much easier to cover along the fiber surface under shearing force, which can form full coverage on the fiber surface. On the other hand, strong $\pi-\pi$ interactions existing between adjacent graphene are beneficial to forming interconnected electron-transport pathways for high electrical conductivity (Balandin et al. 2008).

Calotropis gigantea yarns (CGYs) have received intense attention in recent years due to their unique structure and performance (Zhao et al. 2019). Calotropis gigantea fibers (CGFs) with 80-90\% hollow structures exhibit outstanding hydrophilic or oleophilic properties (Zheng et al. 2017; Chen et al. 2013). Compared to cotton fibers, the CGFs have fewer natural curls in the longitudinal direction, resulting in a smoother and softer surface. By taking advantage of the abovementioned superiorities, the fibers were investigated for applications of reducing the wear of carbon nanotubes, and improving the mechanical strength of polyesters (Ganeshan et al. 2018). Although these studies focused on the properties of CGF-based composites, the functionalization of CGFs by the coating of graphene with large-scale production capacity has not been investigated.

Herein, we report a graphene-coated CGY prepared by pad dyeing, which endows it with not only high conductivity but also scalable production capacity. By optimizing the dyeing parameters such as graphene oxide (GO) concentration, pad dyeing cycles, and reduction time, the graphene-coated CGYs display a high electrical conductivity of $6.9 \mathrm{~S} / \mathrm{m}$. We also compare the dyeing parameters in determining the conductivity of the yarns through range analysis, showing that the electrical conductivity of the graphene-coated CGYs exhibits a strong dependence on the concentration of GO and pad dyeing cycles. The combination between the CGFs and graphene renders the modified CGYs good conducting stability for washing and bending. The high-performance graphene-coated CGYs show great potential in the application of wearable textile-based electronics.

\section{Experimental}

Materials

The GO solution was prepared by the Hummers method using natural graphite from Suzhou Industrial Park Bomeida Reagent Instrument Co., Ltd. The CGYs were supplied by Yixing Zhongchang Yarndyed Co., Ltd. Analytical-grade sodium, hydrochloric acid, and potassium permanganate $\left(\mathrm{KMnO}_{4}\right)$ reagents were purchased from Sinopharm Chemical Reagent Co., Ltd, China. Concentrated sulfuric acid, hydrogen peroxide at a concentration of $30 \%$, and ascorbic acid were purchased from Jiangsu Qiangsheng Functional Chemical Co., Ltd, China, Shanghai Lingfeng Chemical Reagent Co., Ltd, China, and Suzhou Ketong Biomedical Technology Co., Ltd, China respectively.

\section{Preparation of graphene coated CGYs}

We synthesized the GO by the Hummers method, which was reported in the previous work (Xu et al. 2020; Dwivedi et al. 2021). Briefly, graphite powders (2 g), $\mathrm{NaNO}_{3}\left(2 \mathrm{~g}\right.$ ), and $50 \mathrm{~mL}$ of $\mathrm{H}_{2} \mathrm{SO}_{4}(98 \%$ ) were mixed in a $1000 \mathrm{~mL}$ volumetric flask and kept in an ice bath. The mixture was constantly stirred for $2 \mathrm{~h}$ with the gradual addition of potassium permanganate $(6 \mathrm{~g})$. Then, the ice bath was removed, and the mixture was stirred at $35^{\circ} \mathrm{C}$ until it became pasty brownish and stirred for 2 days. Distilled water was added to the mixture, and then $10 \mathrm{~mL} \mathrm{H}_{2} \mathrm{O}_{2}$ was added to terminate the reaction. For purification, we washed the mixture by rinsing and centrifuging with $10 \% \mathrm{HCl}$ and then deionized (DI) water several times, and the centrifugation speed was approximately $13,000 \mathrm{rpm}$ (Arthi et al. 2015). The CGYs were immersed in GO solution for $2 \mathrm{~h}$ before coating, and then the CGYs passed through a small rolling mill at a speed of approximately $0.1 \mathrm{~m} / \mathrm{s}$ to remove excess GO on the surface. After that, the coated yarns were dried in an oven at $60{ }^{\circ} \mathrm{C}$ for $1 \mathrm{~h}$ to attach GO to the surface of the CGYs. By using the green reducing agent ascorbic acid to reduce GO on the surface of pristine CGYs at $80{ }^{\circ} \mathrm{C}$ for $24 \mathrm{~h}$, we prepared the reduced GO (rGO)-modified CGYs. 
Characterization

We measured the surface hydrophilicity of the CGYs by contact angle measuring equipment (Krüss DSA 100, Germany). Before measurement, the CGYs were arranged on the glass slides, and the distance between adjacent CGYs was $0.28 \mathrm{~mm}$. The tensile properties of the CGYs were tested by a universal material testing machine (INSTRON-3365, Instron, USA), and the length of the CGYs was $10 \mathrm{~cm}$. The electrical properties of the rGO-CGYs were measured using a Keithley DMM7510 instrument. The rGO-CGYs were clamped with copper wire at a distance of $5 \mathrm{~cm}$, and conductive silver paste was used to fix and minimize the contact resistance between the samples and electrodes (Cho et al. 2015). Thermogravimetric analysis (TGA) was performed on a SDT Q600 (simultaneous DSC-TG analyser, TA Instruments, USA). The surface morphologies and elemental distribution of the CGYs were measured using a scanning electron microscope (SEM, Hitachi TM3030, Tokyo, Japan). The surface roughness of the CGYs was measured using atomic force microscopy (AFM, Dimension Loon, Bruker Company, USA). The secondary structure of the CGYs was examined using Fourier transform infrared spectroscopy (FT-IR) spectroscopy (Nicolet 5700, USA). Raman spectra of the CGYs were characterized on a Jobin Yvon LabRam HR800 (632.8 $\mathrm{nm}$ laser). We used the polydimethylsiloxane (PDMS) during bending test to ensure a reasonable result by avoiding the mutual contact of the rGOmodified fiber in the curved shape.

\section{Results and discussion}

\section{Graphene coating onto CGYs}

GO possesses good dispersion stability due to the strong electrostatic repulsion between the charged GO (Kim et al. 2012). Although GO can be easily attached to CGYs by physical bonding, it is difficult to obtain a completely covered structure because of the intrinsic repulsion between GO and the coated yarns. Therefore, we employed a commercialized pad dyeing instrument to accomplish the modification (Fig. 1a). The pristine CGYs were firstly immersed in GO solution to make the fiber fully in contact with the GO flakes. Then such CGYs were treated through a rolling mill to be further coated. After GO modification, we reduced GO into $\mathrm{rGO}$ by transferring the GO-coated yarn into the ascorbic acid solvent (with a weight ratio of $10: 1$ to $\mathrm{GO}$ ) at $80{ }^{\circ} \mathrm{C}$.

Figure $1 \mathrm{~b}$ presents schematic diagrams of pristine CGY, GO-CGY, and rGO-CGY, as well as the images of corresponding yarns. The size distribution of GO in Fig. 1c exhibits the size of the GO flakes is mainly between 2 and $6 \mu \mathrm{m}$, which is obtained from the inserted SEM images. We investigated the GO concentration, pad dyeing cycles, and reduction time in determining the properties of graphene-coated CGYs through an orthogonal design with three factors and three levels, of which sample specifications are shown in Table 1. We show the samples with good volume conductivity (Sample 2, Sample 5, and Sample 8) to present the performance and make comparisons, which are denoted as rGO-CGY-4, rGO-CGY-7, and rGO-CGY-10 respectively. The factors determining the conductivity of yarn were analysed by range analysis with all sample data included. Figure 1d illustrates that the viscosity of the 3,5 , and $7 \mathrm{mg} / \mathrm{mL} \mathrm{GO}$ solutions is $26.6 \pm 4.8,41.4 \pm 7.4$, and $61.6 \pm 7.0 \mathrm{~Pa}$.s respectively, indicating that its viscosity increases as the GO concentration increases.

Surface morphology and elements

Figure 2a, b show the pristine and $\mathrm{rGO}$ modified CGYs, of which surface changes from natural color to black due to the attachment of rGO onto the fiber surface. After modification, the surface elements of the yarns change significantly. Figure $2 \mathrm{c}$ shows the atomic percentages of $\mathrm{C}, \mathrm{N}$, and $\mathrm{O}$ on the surface of pristine CGY, GO-CGY-10, and rGO-CGY-10. The $\mathrm{C}, \mathrm{N}$, and $\mathrm{O}$ of the pristine yarn are $57.780 \%, 4.678 \%$, and $37.542 \%$, respectively, while they become $59.323 \%, 3.133 \%$, and $37.544 \%$ after GO modification. The increase in $\mathrm{C}$ and $\mathrm{O}$ and the decrease in $\mathrm{N}$ can be attributed to the attachment of $\mathrm{GO}$ on the surface of CGY. After reduction, $\mathrm{C}$ increases by $61.855 \%$, and $\mathrm{N}$ and $\mathrm{O}$ decrease by $2.536 \%$ and $35.609 \%$, respectively. The surface element changes indicate that the reduction process successfully facilitates the transformation of $\mathrm{rGO}$ from GO, which is consistent with our previous observation (Cai et al. 2017).

Figure $2 \mathrm{~d}-\mathrm{d}_{2}$ show the surface morphology of pristine CGY, GO-CGY-10, and rGO-CGY-10. The 
Fig. 1 a Schematic diagram of the GO-CGYs through pad dyeing. b Illustrations and photos of the pristine, GO-modified, and rGO-modified CGYs. c The particle size distribution and SEM image (insert) of the prepared GO. d The viscosity of GO solution

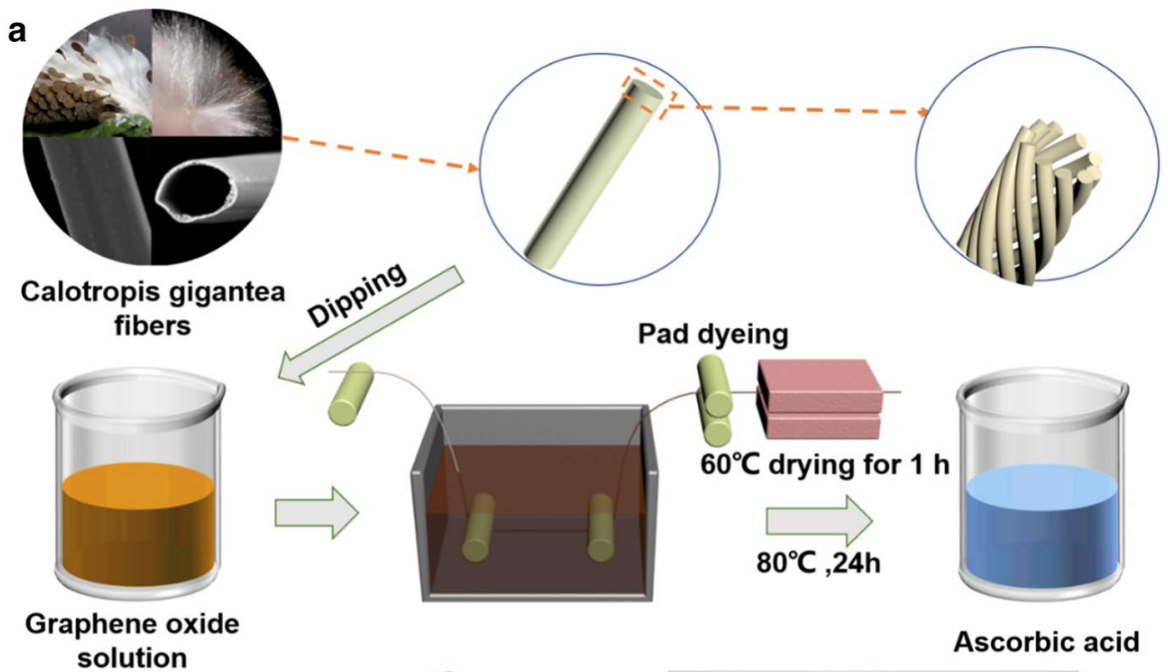

b
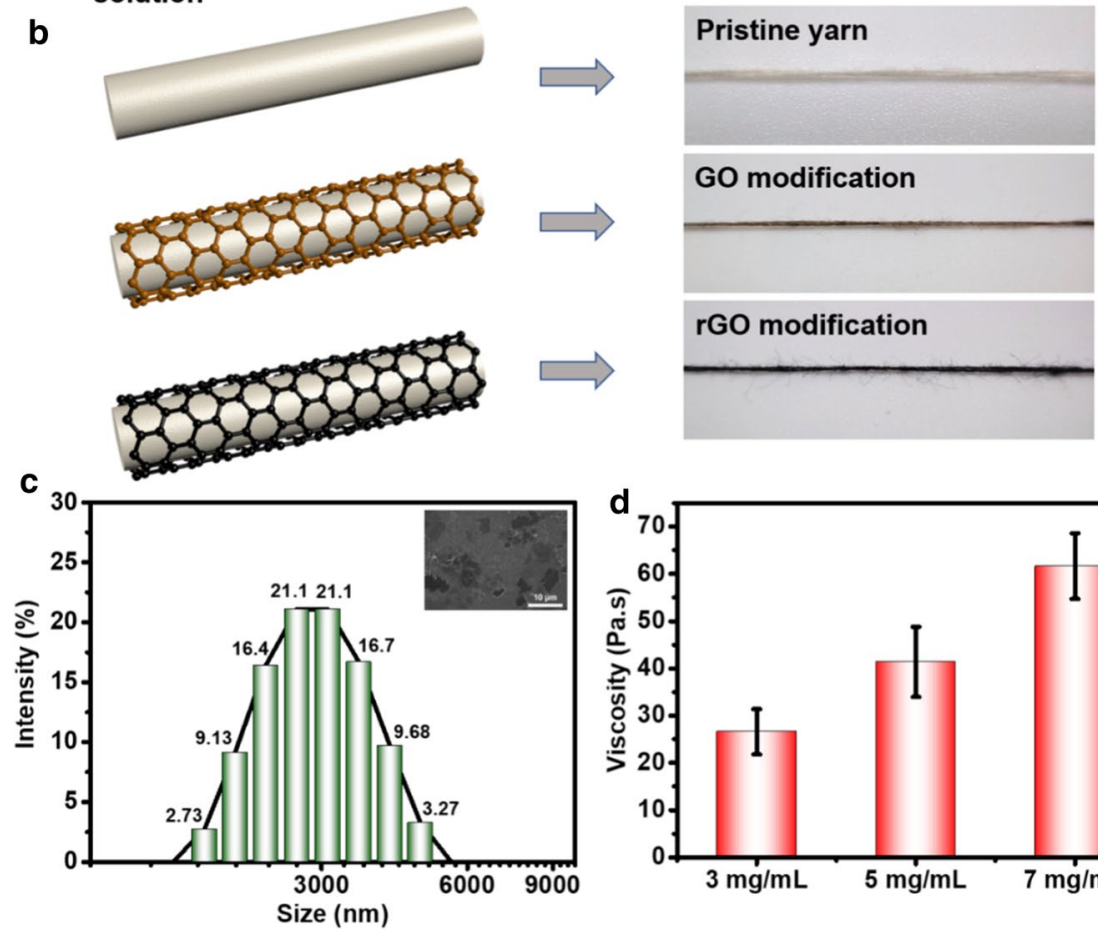

rGO modification

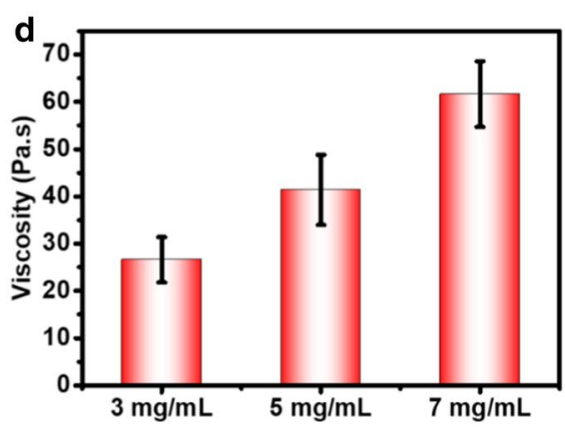

atomic force microscopy images present the surface roughness of the pristine CG fiber and rGO-CG-10 fiber is 51.8 and $155 \mathrm{~nm}$, respectively, showing that the roughness of the CG fibers increases significantly after treatment. Compared to pristine CGYs with smooth surfaces, the GO-CGY-10 shows rough and uneven surfaces due to the existence of GO layers, and the rGO-CGY-10 has a wrinkled and crumpled surface which is similar to graphene fibers (Ding et al. 2016; Abdou et al. 2016; Sheng et al. 2017).
This volume shrinkage is mainly due to intermittent pad dyeing and dehydration shrinkage (Chen et al. 2015). It is beneficial to compacting the rGO coatings into a dense structure and forming a strong interaction between the rGO and the CGYs (Park et al. 2015). Ascorbic acid, as a green reducing agent, promotes the reduction of GO on the surface of pristine CGY without observable damage to the yarn (Zhang et al. 2010). Figure $2 \mathrm{e}-\mathrm{e}_{2}, \mathrm{f}-\mathrm{f}_{2}$, and $\mathrm{g}-\mathrm{g}_{2}$ show the surface element distributions of the pristine CGY, 
Table 1 The specifications of rGO-CGYs through pad dyeing

\begin{tabular}{llllll}
\hline Sample & $\begin{array}{l}\text { Additive } \\
\text { concentra- } \\
\text { tion } \\
(\mathrm{mg} / \mathrm{mL})\end{array}$ & $\begin{array}{l}\text { Reduction } \\
\text { the }\end{array}$ & $\begin{array}{l}\text { Pad dyeing } \\
\text { cycles }\end{array}$ & $\begin{array}{l}\text { Contact angle } \\
\left({ }^{\circ}\right)\end{array}$ & $\begin{array}{l}\text { Volume conductivities } \\
(\mathrm{S} / \mathrm{m})\end{array}$ \\
\hline 1 & 3 & 12 & 4 & $133.83 \pm 5.12$ & $0.29 \pm 0.08$ \\
2 & 5 & 36 & 4 & $147.47 \pm 5.21$ & $1.99 \pm 0.35$ \\
3 & 7 & 24 & 4 & $135.23 \pm 4.08$ & $0.56 \pm 0.12$ \\
4 & 3 & 24 & 7 & $139.03 \pm 8.49$ & $0.33 \pm 0.05$ \\
5 & 5 & 12 & 7 & $150.27 \pm 4.94$ & $3.50 \pm 0.44$ \\
6 & 7 & 36 & 7 & $145.03 \pm 2.97$ & $0.98 \pm 0.09$ \\
7 & 3 & 36 & 10 & $143.83 \pm 5.00$ & $0.74 \pm 0.05$ \\
8 & 5 & 24 & 10 & $150.10 \pm 3.52$ & $6.90 \pm 0.19$ \\
9 & 7 & 12 & 10 & $142.33 \pm 5.87$ & $1.40 \pm 0.22$ \\
\hline
\end{tabular}

GO-CGY-10, and rGO-CGY-10, respectively. The surface morphology and element distribution indicate that graphene uniformly covers the CGFs, which is of great significance to both the conductivity and stability of the yarn.

Figure 3a shows the Raman spectra of the GO and rGO-modified CGYs. The D-band at $1370 \mathrm{~cm}^{-1}$ (related to defects or heteroatom-doping), and G-band at $1590 \mathrm{~cm}^{-1}$ (attributed to the vibrations of the $s p^{2}$ bonded crystalline carbon) indicate that graphene wraps onto the CGYs. The GO-CGY shows a higher $\mathrm{D}$ band, while the rGO-CGY shows a higher $\mathrm{G}$ band, which can be seen in the high-resolution fitting spectrum of Raman spectra of GO-CGY (Fig. 3b), and the high-resolution fitting spectrum of Raman spectra of rGO-CGY (Fig. 3c). The intensity ratio of the D-band and G-band increases from 0.9 to 1.2 and the full width at half maximum (FWHM) of the D-band decreases from 71 to 53 (Fig. 3d). The enhancement of the D-band is mainly attributed to the formation of small-sized $s p^{2}$ domains (disordered carbon) during the reduction process. The decrease in the FWHM suggests an increase in the structural order. These changes show that the GO-CGYs are chemically converted into rGO-CGYs, which is consistent with the observation of graphene-coated elastic wearable electronics reported in the literature (Wang et al. 2017).

Figure $3 \mathrm{e}$ shows changes in the surface wettability of the pristine CGYs and the modified CGYs. The contact angle of the pristine CGYs is approximately $20^{\circ}$ (droplets drop at $1 \mathrm{~s}$ ). After functionalization, the contact angle greatly increases to $150^{\circ}$ (the rGO-CGY) and the contact angle of the rGO-CGYs remains stable after $600 \mathrm{~s}$. With rGO modification for 4 pad dyeing cycles, the contact angle reaches $145^{\circ}$. With increasing pad dyeing, the contact angle increases slowly up to $150^{\circ}$ after 10 cycles. The increase in the surface contact angle is mainly due to the changes in surface groups and surface structure. Pristine CGY is a hydrophilic material (Zheng et al. 2017), while graphene is a hydrophobic material (Zhang et al. 2014). The oxygen-related functional groups on the surface of the CGYs were eliminated after the GO was reduced, resulting in a significant increase in the hydrophobicity of the CGYs (Bhattacharjee et al. 2020). On the other hand, graphene coating also increases the surface roughness of the CGY, resulting in high hydrophobicity (Chen et al. 2016).

Figure 3f shows the thermal stability of the CGYs. The CGYs experience a weight loss as the temperature increases. When the temperature rises from 20 to $700{ }^{\circ} \mathrm{C}$, the corresponding weight loss reaches approximately $85 \%$. The small amount of loss within $100{ }^{\circ} \mathrm{C}$ can be attributed to moisture evaporation. At approximately $400{ }^{\circ} \mathrm{C}$, rGO begins to decompose, and the rGO-CGY-10 shows a more obvious weight loss. The loss should be attributed to the thermal-induced destruction in the CGYs considering that graphene has remarkable temperature stability and tolerance.

\section{Hydrophilicity and mechanical properties}

The typical tensile strain curves of pristine and rGO-modified CGYs in Fig. 4a exhibit that the graphene coating can result in a higher breaking strength and a lower elongation as well as a higher modulus. The breaking strength of the pristine 
Fig. 2 a The image of the pristine CGYs. b The image of the rGO-CGY-10. $\mathbf{c}$ The surface element content of the CGYs, the surface morphology, and the atomic force microscopy images of d the pristine CGY, $\mathbf{d}_{1} \mathrm{GO}$ CGY-10, $\mathbf{d}_{2}$ rGO-CGY-10, and $\mathbf{e}-\mathbf{e}_{2} \mathrm{C}, \mathrm{N}$, and $\mathrm{O}$ distributions of pristine CGY, $\mathbf{f}-$ $\mathbf{f}_{2} \mathrm{C}, \mathrm{N}$, and $\mathrm{O}$ distributions of the GO-CGY-10, and g- $\left.-\mathrm{g}_{2}\right) \mathrm{C}, \mathrm{N}$, and $\mathrm{O}$ distributions of the rGO-CGY-10
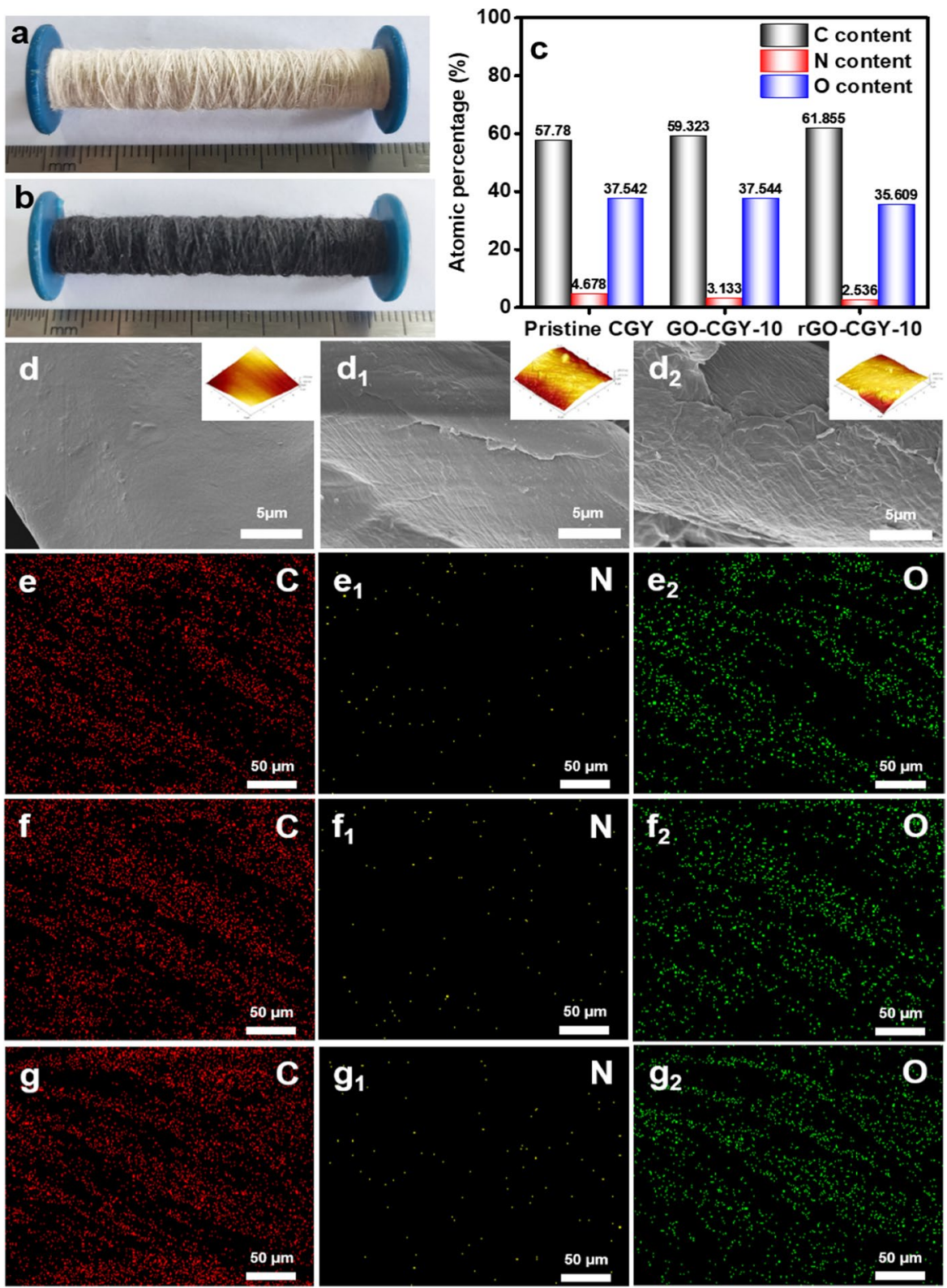

CGYs is approximately $168 \mathrm{cN}$, and it gradually increases with the enhancement of dyeing cycles (Fig. 4b). After 10 cycles of dyeing, the breaking strength finally becomes $195 \mathrm{cN}$. The increase in breaking strength may be due to the high mechanical strength of graphene (Geim and Novoselov 2007). The attachment of graphene on the fiber surface promotes a stronger combination between fibers (Mackin et al. 1992). Figure 4c shows that the increase in dyeing cycles slightly decreases the tensile strain of the CGYs, of which tensile strain is
7.2\% and $4.1 \%$ for pristine CGYs and rGO-CGY-10, respectively.

Figure $4 \mathrm{~d}$ shows the electrical resistance of the CGYs after graphene coating. The resistance of the rGO-CGY drastically decreases with the increase of dyeing cycles, where it is approximately $800 \mathrm{k} \Omega$ and $210 \mathrm{k} \Omega$ for the rGO-CGY-4 and rGO-CGY-10, respectively. The calculated electrical conductivity of the rGO-CGYs is shown in Fig. 4e. The formula of electrical conductivity of the CGYs is $\mathrm{K}=\mathrm{RS} / \mathrm{L}$ ( $\mathrm{K}$ is electrical conductivity, $\mathrm{R}$ is electrical resistance, 
Fig. 3 a Raman spectra of the CGYs after functionalization. b The high-resolution spectrum of Raman spectra of the GO-CGY-10. c The high-resolution spectrum of the Raman spectra of the rGO-CGY-10. d The intensity ratios of the D-band and G-band and the FWHM of the D-band for the GO-CGY-10 and rGO-CGY-10. e The contact angle of the pristine CGYs after graphene coating. $f$ The TGA curves of graphene-coated CGYs
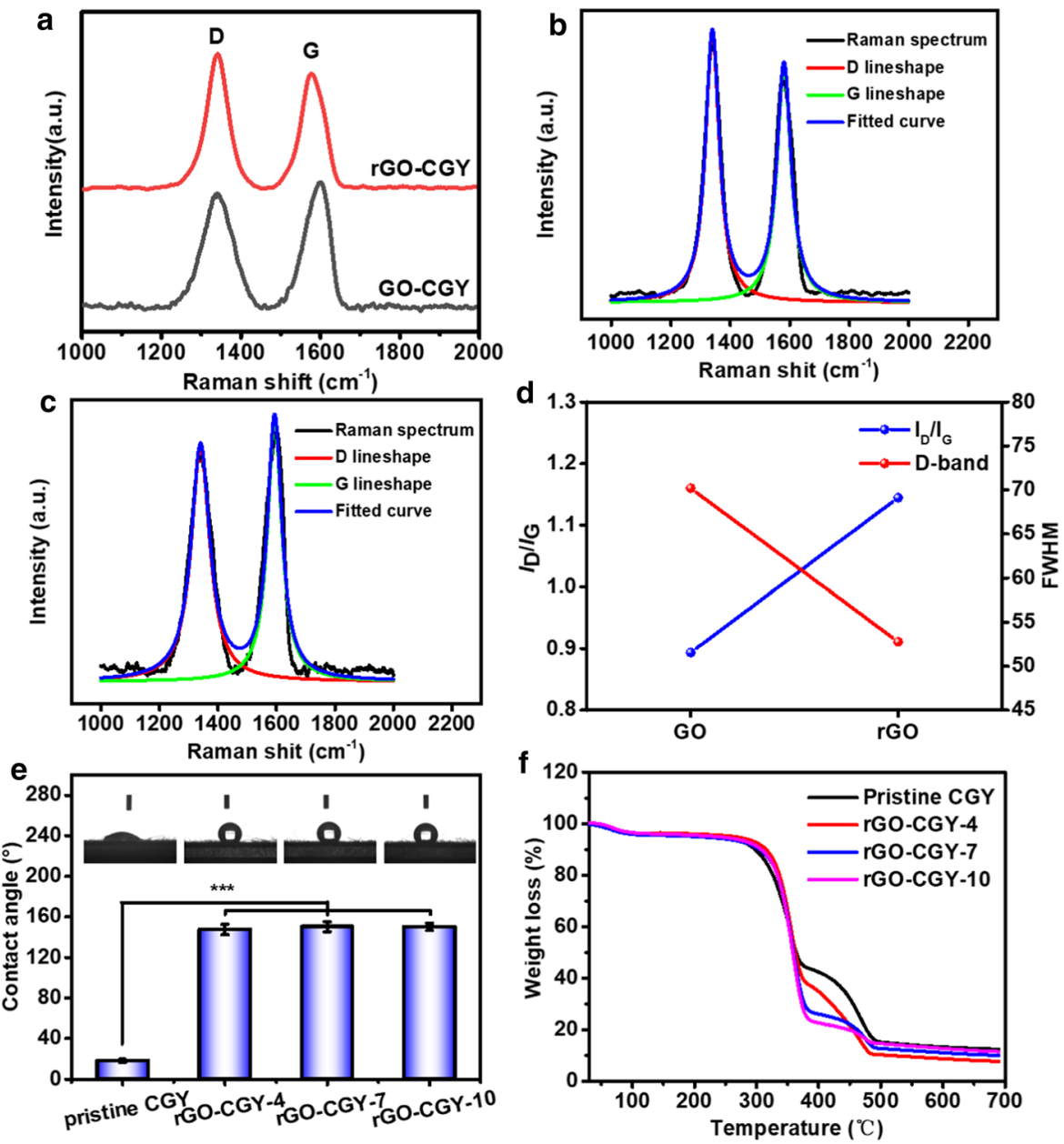

$\mathrm{S}$ is the cross-sectional area, and 1 is the length of CGY). Compared to the electrical conductivity of the rGO-CGY-4, the electrical conductivity of the rGO-CGY-10 is improved. It exhibits a high electrical conductivity of $6.9 \mathrm{~S} / \mathrm{m}$, while the rGO-CGY-4 only presents a volume conductivity of $2 \mathrm{~S} / \mathrm{m}$. With the combination of graphene, the strong $\pi-\pi$ interactions between adjacent graphene form interconnected electron-transport pathways. With more pad dyeing cycles, the rGO-CGY-10 holds more electron-transport pathways and obtains high electrical conductivity.

The range analysis of the coating parameters in determining the electrical conductivity of rGOCGYs in Fig. 4f shows that the range value of the additive concentration of GO solution, the reduction time, and the pad dyeing cycles is $3.665,1.344$, and 2.048 , respectively. The electrical conductivity of the
rGO-CGYs largely depends on the additive concentration of GO solution and pad dyeing cycles, while slightly relies on the reduction time. When the concentration of GO solution is $3 \mathrm{mg} / \mathrm{mL}$, the content of graphene is low, while when the concentration of GO solution is $7 \mathrm{mg} / \mathrm{mL}$, the viscosity of graphene solution is too high to adhere to the surface of CGY. It leads to fewer electron-transport pathways. The degree of graphene coating on the yarn surface can be augmented with the increase in pad dyeing cycles, which can also increase the number of electron transport pathways on the surface of the CGYs.

Figure 5a presents the FTIR spectra of the pristine CGY, GO-CGY-10, and rGO-CGY-10. The FTIR spectrum of pristine CGY displays peaks at approximately $3340 \mathrm{~cm}^{-1}, 2900 \mathrm{~cm}^{-1}, 1645 \mathrm{~cm}^{-1}$, $1430 \mathrm{~cm}^{-1}$ and $1060 \mathrm{~cm}^{-1}$, which are mainly attributed to $\mathrm{OH}$ stretching, asymmetrical $\mathrm{C}-\mathrm{H}$ stretching, 
Fig. 4 a The mechanical curves of the CGYs. b The breaking strength of the graphene-coated CGYs. c The tensile strain of the graphene-coated CGYs. d The electrical resistance of the rGO-CGYs. e The volume electrical conductivity of the rGO-CGYs. f Range analysis results of the GO concentration, reduction time, and pad dye times in determining the conductivity
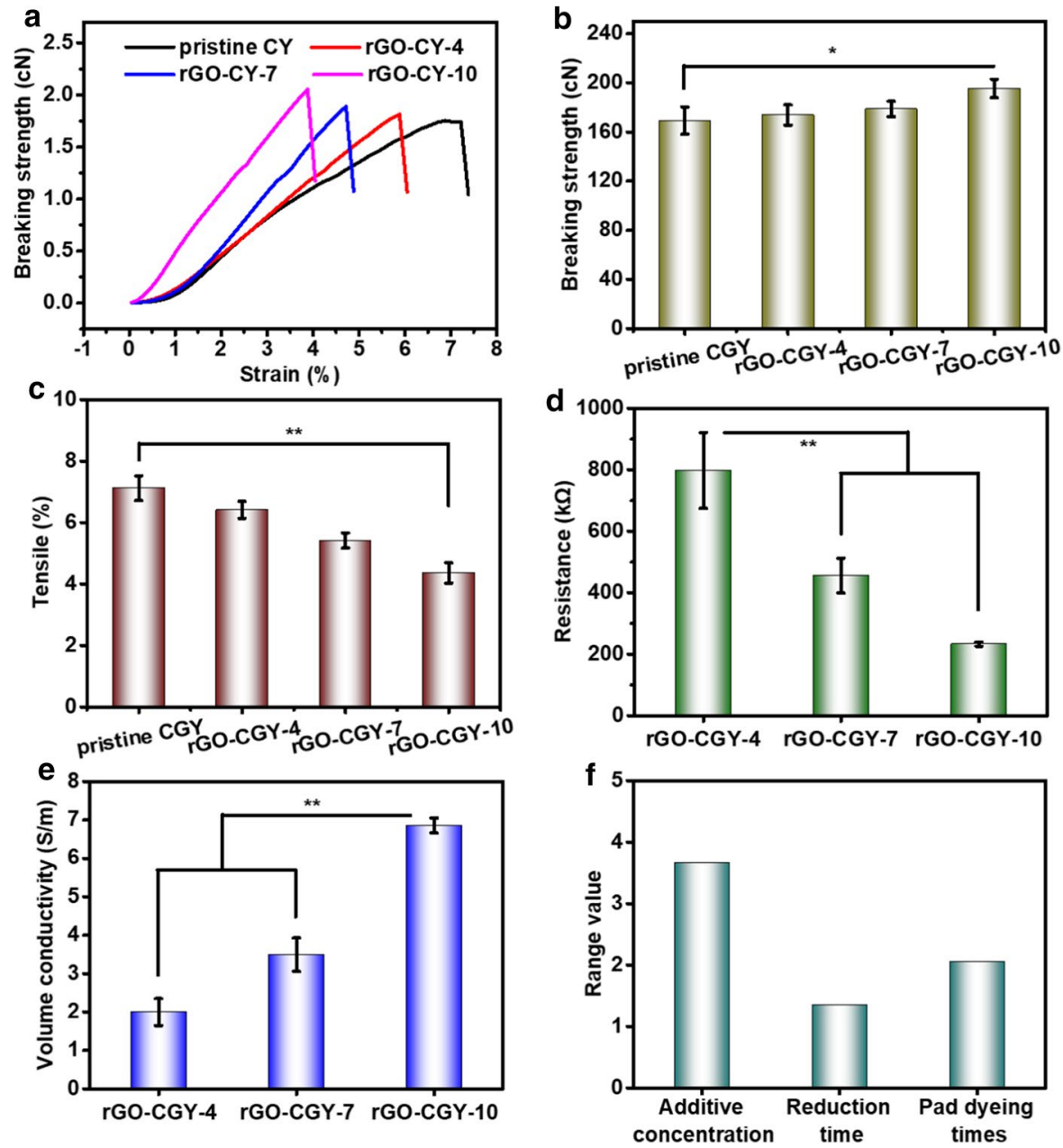

$\mathrm{C}=\mathrm{O}$ stretching, $\mathrm{CH}_{2}$ symmetrical bending and $\mathrm{C}-\mathrm{O}$ stretching, respectively (Xu et al. 2015). After graphene coating, no evident change is found in the FTIR spectrum of the GO-CGY-10, which is consistent with the previous research (Xu et al. 2015). However, the FTIR spectrum of the rGO-CGY-10 changed obviously after ascorbic acid treatment. The peak at $1645 \mathrm{~cm}^{-1}$ disappears and the peaks at $2900 \mathrm{~cm}^{-1}$ and $3340 \mathrm{~cm}^{-1}$ become weaker, which implies that $\mathrm{GO}$ on the surface of CGY is successfully reduced to rGO after the treatment ( $\mathrm{Xu}$ et al. 2015).

Figure $5 \mathrm{~b}$ shows that the characteristic absorption wavenumbers of intramolecular hydrogen bonds $\mathrm{O}(2) \mathrm{H} \cdots \mathrm{O}(6), \mathrm{O}(3) \cdots \mathrm{O}(5)$, and $\mathrm{O}(6) \cdots \mathrm{O}\left(3^{\prime}\right)$ in CGYs are $3310-3233 \mathrm{~cm}^{-1}, 3375-3340 \mathrm{~cm}^{-1}$, and $3455-$ $3410 \mathrm{~cm}^{-1}$, which are named I, II, and III respectively (Oh et al. 2005). The percentage of I changes slightly from 61.32 to $64.74 \%$, the percentage of II increases moderately from 25.14 to $31.97 \%$, and the percentage of III decreases significantly from 13.54 to $3.29 \%$. It reveals that the content of III in the CGY decreases during the treatment. The CGYs consisting of celluloses are rich in positively charged hydrogen, while GO has many negative groups such as carboxyl and hydroxyl groups (Oh et al. 2005; Ling et al. 2013). We postulate a bonding mechanism between the CGYs and the rGO as well as glucose residues are designated as the repeating unit of CGYs (French 2017), which is shown in Fig. 5c. Pristine CGY is coated with GO, and ascorbic acid is used to convert GO into rGO. The negatively charged GO species and positively charged hydrogen in CGY are combined by electrostatic attraction, which is consistent with reported work (Dashairya et al. 2018). The spacing between graphene layers is similar to the thickness of one water molecule, which also allows hydrogen 
Fig. 5 a FTIR spectra of the pristine CGY, GOCGY-10, and rGO-CGY-10. b Proportion of hydrogen bonds in infrared peaks of the pristine $\mathrm{CGY}$, GOCGY-10, and rGO-CGY-10. c Illustration of bonding mechanism between the pristine CGY and graphene
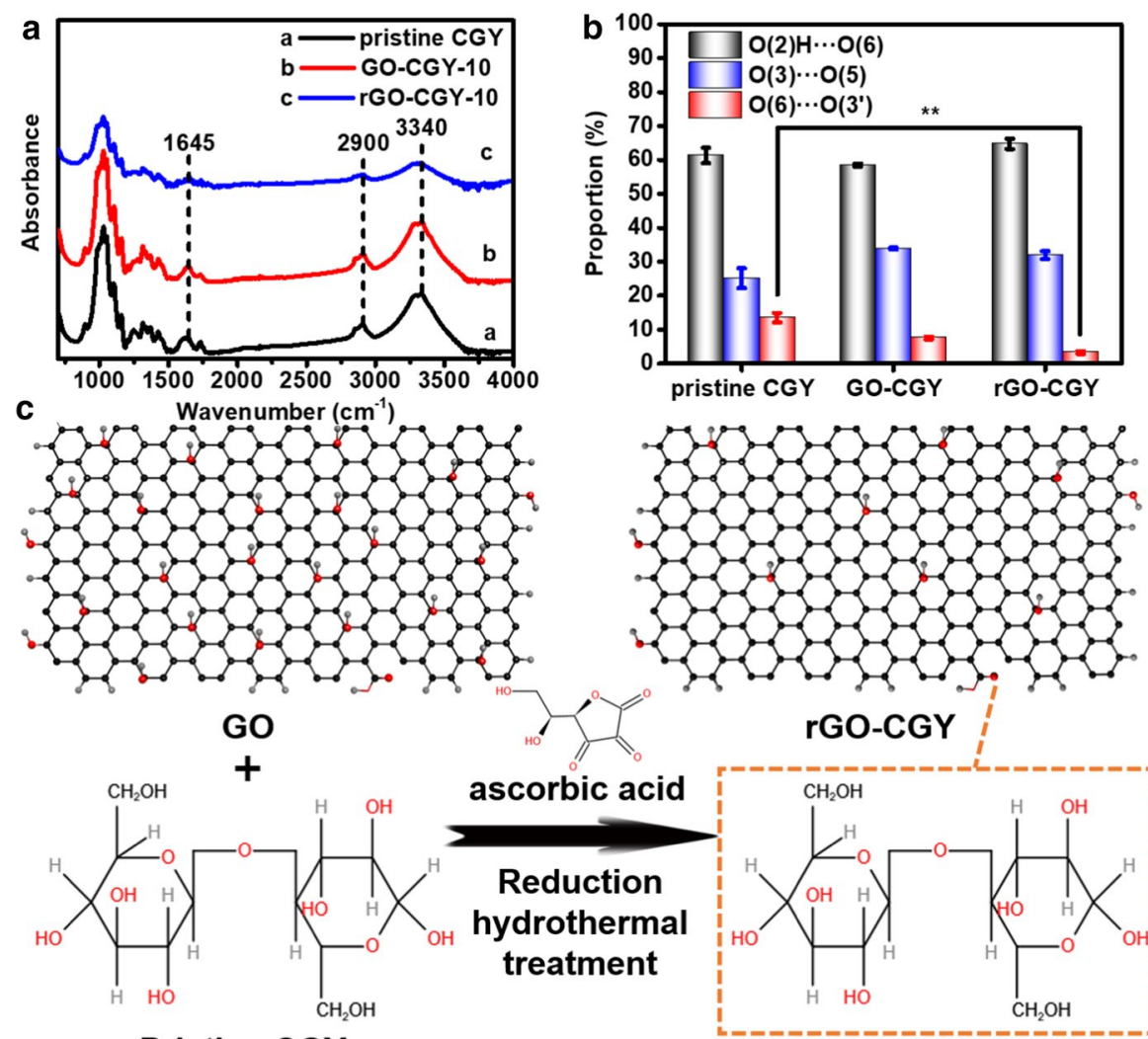

Pristine CGY bonding between the GO and CGFs (Dikin et al. 2007). Such postulation can also be supported by the FTIR and EDS results. The atomic percentage of $\mathrm{O}$ not only does not increase obviously after GO coating but also decreases significantly after reduction. Meanwhile, the content of III in CGYs greatly drops during the whole process.

To evaluate the washability of the graphene-functionalized CGY, we washed the rGO-CGY-10 with DI water and laundry liquid. After being washed by DI water, the volume conductivity of the rGO-CGY-10 slightly decreases with increasing washing time (Fig. 6a). Similarly, the volume conductivity shows a similar trend when the yarn is washed in laundry liquid (Fig. 6b). The yarns still maintain very good conductivity after washing in both DI water and laundry liquid, showing the stability of the coated graphene layers. Figure $6 \mathrm{c}-\mathrm{c}_{2}$ show the photos of the bending process of the rGO-CGY-10. Figure $6 \mathrm{~d}$ and e present the resistance change of the rGO-CGY-10 during cyclic bending from 0 to $50 \%$ and 0 to $75 \%$ respectively. The bending was calculated by the distance change/initial length of CGYs during the bending test. We show the resistance change with $\left(\mathrm{R}-\mathrm{R}_{0}\right) / \mathrm{R}_{0}$, where $R_{0}$ and $R$ refer to the initial resistance and the resistance under bending, respectively. The resistance of the yarn slightly increases from 255 to $262 \mathrm{k} \Omega$ and the bending ranges from 0 to $50 \%$. The resistance change is very stable throughout the cyclic bending process, and the relative resistance change is as low as $3.2 \%$. Even though under high-degree bending up to $75 \%$, the resistance change is still very minor (4.3\%). The CGY has a very limited response to bending, showing great potential in flexible wearable electronic devices.

\section{Conclusions}

In summary, we have reported a conductive CGY through pad dyeing with graphene, showing mass production capacity and high conductivity. When the GO concentration and pad dyeing cycles reach $5 \mathrm{mg} /$ $\mathrm{mL}$ and 10 times, respectively, the graphene-coated 
Fig. 6 a The volume electrical conductivity changes of the rGO-CGY-10 with DI water washing. b The volume electrical conductivity changes of the rGO-CGY-10 with laundry liquid washing. $\mathbf{c}-\mathbf{c}_{2}$ Photos of the rGO-CGY-10 at initial length, $50 \%$ bending, and $75 \%$ bending. $\mathbf{d}$ The electrical response of the rGO-CGY-10 under cyclic bending from 0 to $50 \%$. e The electrical response of the rGO-CGY-10 under cyclic bending from 0 to $75 \%$ a
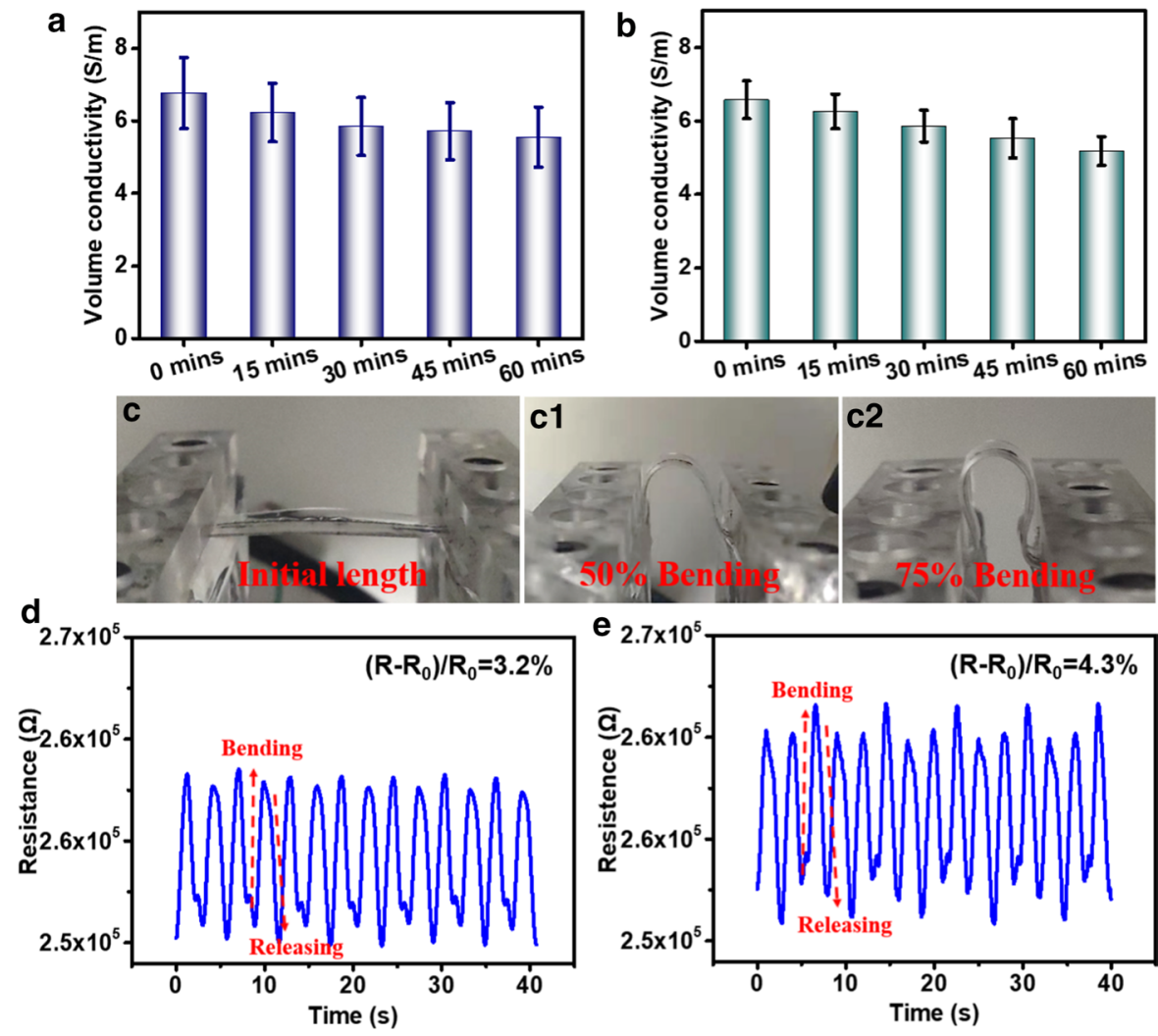

b 0 mins 15 mins $_{30}$ mins 45 mins 60 mins
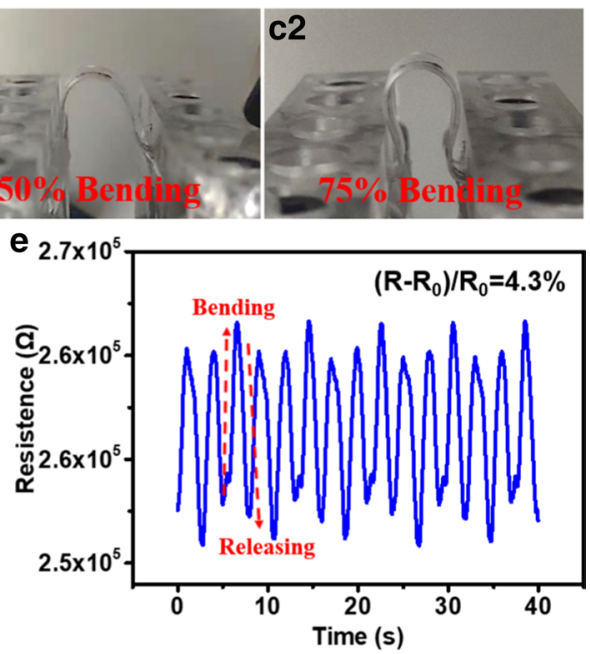

CGYs exhibit high electrical conductivity and better breaking strength. The range analysis shows that the electrical conductivity of the graphene-coated CGYs exhibits a strong dependence on both the concentration of GO and dyeing cycles and a very marginal dependence on the reduction time. This coating approach promotes the formation of the hydrogenoxygen bond between CGFs and graphene, endowing the functionalized CGYs with good conductive stability to not only washing but also bending. Particularly, pad dyeing for preparing conductive CGYs can be rapidly extended to almost any other yarns, paving a new way for developing large-scale production, lowcost, and high-performance conductive yarns.

Acknowledgments This work was financially supported by the National Key R\&D Program of China (2021YFE0111100), the Shanghai Science and Technology Committee (21015800600), the "Top six talent peaks" program of Jiangsu (GDZB-035) and the Science and Technology Project of Nantong (JC2020082). We would like to thank the China National Textile and Apparel Council (J202002), Jiangsu Provincial Government Scholarship Program and Joint Scientific Research Project of Sino-Foreign Cooperative Education Platform of Jiangsu Higher Education Institutions (5011500720) and Projects with Numbers FZ20190257, XJFZ/2021/7 and 2021fx010104 for their support.

Funding The authors have not disclosed any funding.

\section{Declarations}

Conflict of interest The authors have nothing to disclose regarding the conflict of interest.

Open Access This article is licensed under a Creative Commons Attribution 4.0 International License, which permits use, sharing, adaptation, distribution and reproduction in any medium or format, as long as you give appropriate credit to the original author(s) and the source, provide a link to the Creative Commons licence, and indicate if changes were made. The images or other third party material in this article are included in the article's Creative Commons licence, unless indicated otherwise in a credit line to the material. If material is not included in the article's Creative Commons licence and your intended use is not permitted by statutory regulation or exceeds the permitted use, you will need to obtain permission directly from the copyright holder. To view a copy of this licence, visit http://creativecommons.org/licenses/by/4.0/. 


\section{References}

Abdou JP, Reynolds KJ, Pfau MR, van Staden J et al (2016) Interfacial crystallization of isotactic polypropylene surrounding macroscopic carbon nanotube and graphene fibers. Polymer 91:136-145

Arthi G, Paulchamy B, Bd LJJ (2015) A simple approach to stepwise synthesis of graphene oxide nanomaterial. J Nanomed Nanotechnol 06(01):2157-7439

Balandin AA, Ghosh S, Bao WZ, Calizo I et al (2008) Superior thermal conductivity of single-layer graphene. Nano Lett 8(3):902-907

Bhattacharjee S, Macintyre CR, Bahl P, Kumar U et al (2020) Reduced graphene oxide and nanoparticles incorporated durable electroconductive silk fabrics. Adv Mater Interfaces 7(20):2000814

Cai GM, Xu ZL, Yang MY, Tang B et al (2017) Functionalization of cotton fabrics through thermal reduction of graphene oxide. Appl Surf Sci 393:441-448

Chen Q, Zhao T, Wang M, Wang J (2013) Studies of the fibre structure and dyeing properties of Calotropis gigantea, kapok and cotton fibres. Color Technol 129(6):448-453

Chen SH, Ma WJ, Cheng YH, Weng Z et al (2015) Scalable non-liquid-crystal spinning of locally aligned graphene fibers for high-performance wearable supercapacitors. Nano Energy 15:642-653

Chen PY, Sodhi J, Qiu Y, Valentin TM et al (2016) Multiscale graphene topographies programmed by sequential mechanical deformation. Adv Mater 28(18):3603-3603

Cho SY, Yun YS, Lee S, Jang D et al (2015) Carbonization of a stable $\beta$-sheet-rich silk protein into a pseudographitic pyroprotein. Nature 6:7145

Dashairya L, Rout M, Saha P (2018) Reduced graphene oxidecoated cotton as an efficient absorbent in oil-water separation. Adv Compos Hybrid Mater 1(1):135-148

Dikin DA, Stankovich S, Zimney EJ, Piner RD et al (2007) Preparation and characterization of graphene oxide paper. Nature 448(7152):457-460

Ding XT, Bai J, Xu T, Li CX et al (2016) A novel nitrogendoped graphene fiber microelectrode with ultrahigh sensitivity for the detection of dopamine. Electrochem Commun 72:122-125

Dwivedi UK, Singh S, Shukla SC, Hashmi SAR (2021) Investigation on tribo-characteristics of Calotropis gigantea fiber-reinforced-CNT modified polymer composites. Polym Bull 2021: 1-11

French AD (2017) Glucose, not cellobiose, is the repeating unit of cellulose and why that is important. Cellulose 24:4605-4609

Gandla S, Naqi M, Lee MG, Lee JJ et al (2020) Highly linear and stable flexible temperature sensors based on laserinduced carbonization of polyimide substrates for personal mobile monitoring. Adv Mater Technol 5(7):2000014

Ganeshan P, Kumaran SS, Raja K, Venkateswarlu D (2018) An investigation of mechanical properties of madar fiber reinforced polyester composites for various fiber length and fiber content. Mater Res Express 6(1):015303

Geim AK, Novoselov KS (2007) The rise of graphene. Nat Mater 6(3):183-191
Jia LC, Xu L, Ren F, Ren PG et al (2019) Stretchable and durable conductive fabric for ultrahigh performance electromagnetic interference shielding. Carbon 144:101-108

Kim J, Cote LJ, Huang JX (2012) Two dimensional soft material: new faces of graphene oxide. Acc Chem Res 45(8):1356-1364

Lin ZM, Yang J, Li XS, Wu YF et al (2018) Large-scale and washable smart textiles based on triboelectric nanogenerator arrays for self-powered sleeping monitoring. Adv Funct Mater 28(1):1704112

Ling S, Fugetsu, B J M L (2013) Mass production of graphene oxide from expanded graphite. Mater Lett 109(1):207-210

Liu ZK, Zheng Y, Jin L, Chen KL et al (2021a) Highly breathable and stretchable strain sensors with insensitive response to pressure and bending. Adv Funct Mater 31(14):2007622

Liu ZK, Chen KL, Fernando A, Gao Y et al (2021b) Permeable graphited hemp fabrics-based, wearing-comfortable pressure sensors for monitoring human activities. Chem Eng J 403:126191

Liu Z, Li Z, Zhai H, Jin L et al (2021c) A highly sensitive stretchable strain sensor based on multi-functionalized fabric for respiration monitoring and identification. Chem Eng J 426:130869

Lu Y, Sun H, Cheng J, Myong J et al (2020) High performance flexible wearable strain sensor based on rGO and AgNWs decorated PBT melt-blown non-woven fabrics. Sens Actuators A 315:112174

Mackin TJ, Warren PD, Evans AG (1992) Effects of fiber roughness on interface sliding in composites. Acta Metall Mater 40(6):1251-1257

Molina J (2016) Graphene-based fabrics and their applications: a review. RSC Adv 6(72):68261-68291

Oh SY, Yoo DI, Shin Y, Kim HC et al (2005) Crystalline structure analysis of cellulose treated with sodium hydroxide and carbon dioxide by means of X-ray diffraction and FTIR spectroscopy. Carbohydr Res 340(15):2376-2391

Ozen MS, Sancak E, Soin N, Shah TH et al (2018) Unprecedented electromagnetic shielding effectiveness of lightweight nonwoven Ag/PA66 fabrics. Fibers Polym 19(2):321-330

Park SK, Seong CY, Piao Y (2015) A simple dip-coating approach for preparation of three-dimensional multilayered graphene-metal oxides hybrid nanostructures as high performance lithium-ion battery electrodes. Electrochim Acta 176:1182-1190

Ren F, Song DP, Li Z, Jia LC et al (2018) Synergistic effect of graphene nanosheets and carbonyl iron-nickel alloy hybrid filler on electromagnetic interference shielding and thermal conductivity of cyanate ester composites. J Mater Chem C 6(6):1476-1486

Sheng LZ, Wei T, Liang Y, Jiang LL et al (2017) Ultra-high toughness all graphene fibers derived from synergetic effect of interconnected graphene ribbons and graphene sheets. Carbon 120:17-22

Wang CY, Zhang MC, Xia KL, Gong XQ et al (2017) Intrinsically stretchable and conductive textile by a scalable process for elastic wearable electronics. ACS Appl Mater Interfaces 9(15):13331-13338

Wang YM, Zhang LW, Zhang ZW, Sun PY et al (2020) Highsensitivity wearable and flexible humidity sensor based on 
graphene oxide/non-woven fabric for respiration monitoring. Langmuir 36(32):9443-9448

Won C, Lee S, Jung HH, Woo J et al (2020) Ultrasensitive and stretchable conductive fibers using percolated Pd nanoparticle networks for multisensing wearable electronics: crack-based strain and H-2 sensors. ACS Appl Mater Interfaces 12(40):45243-45253

Xu LL, Guo MX, Liu S, Bian S W, J R A (2015) Graphene/ cotton composite fabrics as flexible electrode materials for electrochemical capacitors. RSC Adv 5(32):25244-25249

Xu LL, Liu ZK, Zhai H, Chen X et al (2020) Moisture-resilient graphene-dyed wool fabric for strain sensing. ACS Appl Mater Interfaces 12(11):13265-13274

Zhang JL, Yang HJ, Shen GX, Cheng P et al (2010) Reduction of graphene oxide via L-ascorbic acid. Chem Commun 46(7):1112-1114

Zhang GJ, Guo XX, Wang SL, Wang XL et al (2014) New graphene fiber coating for volatile organic compounds analysis. J Chromatogr B: Anal Technol Biomed Life Sci 969:128-131
Zhao ZY, Zheng ZZ, Chen P, Zhang HF et al (2019) Pretreatment o fCalotropis gigantea fibers with functional plasticizing and toughening auxiliary agents. Text Res J 89(19-20):3997-4006

Zhao Y, Ren M, Shang Y, Li J et al (2020) Ultra-sensitive and durable strain sensor with sandwich structure and excellent anti-interference ability for wearable electronic skins. Compos Sci Technol 200(9):108448

Zheng Y, Cao EJ, Tu LX, Wang AQ et al (2017) A comparative study for oil-absorbing performance of octadecyltrichlorosilane treated Calotropis gigantea fiber and kapok fiber. Cellulose 24(2):989-1000

Publisher's Note Springer Nature remains neutral with regard to jurisdictional claims in published maps and institutional affiliations. 